\title{
Gibbons-Hawking Effect in the Sonic de Sitter Space-Time of an Expanding Bose-Einstein-Condensed Gas
}

\author{
Petr O. Fedichev ${ }^{1,2}$ and Uwe R. Fischer ${ }^{1}$ \\ ${ }^{1}$ Leopold-Franzens-Universität Innsbruck, Institut für Theoretische Physik, Technikerstrasse 25, A-6020 Innsbruck, Austria \\ ${ }^{2}$ Russian Research Center Kurchatov Institute, Kurchatov Square, 123182 Moscow, Russia
}

\begin{abstract}
We propose an experimental scheme to observe the Gibbons-Hawking effect in the acoustic analog of a 1+1-dimensional de Sitter universe, produced in an expanding, cigar-shaped Bose-Einstein condensate. It is shown that a two-level system created at the center of the trap, an atomic quantum dot interacting with phonons, observes a thermal Bose distribution at the de Sitter temperature.
\end{abstract}

Cosmology in the early universe is a branch of physics which is, for all too obvious reasons, removed far from experiment. To use a concise formulation of the primary dilemma which cosmology faces, there exists nothing like the possibility of "reproducing" experiments, because there has in fact "only ever been one experiment, still running, and we are latecomers watching from the back" [1]. While there can be no truly experimental cosmology, as regards in particular the reproduction of the (presumed) extreme conditions which prevailed in the early stages of the universe, one might look for analogous phenomena in condensed matter experiments, which are indeed reproducible, and can be done at energy scales smaller by many orders of magnitude [2]

An archetypical model of cosmology is the de Sitter universe, in which space is essentially empty, and the curvature of space-time is due to a nonvanishing cosmological constant $\Lambda$ 3]. The de Sitter space-time is used in inflationary models of the cosmos [4], displays a cosmological horizon, and is associated with a thermal spectrum of particles at a temperature $T_{\mathrm{dS}} \propto \sqrt{\Lambda}[5]$.

Atomic Bose-Einstein condensates (BECs) [6] have emerged as one of the most suitable condensed matter systems for the simulation of quantum phenomena in effective space-times [7, 8, 9, 10, 11, 12]. In the present study, we investigate an effective $1+1-$ dimensional $(1+1 \mathrm{D})$ de Sitter universe and the associated thermal phonon spectrum of the Gibbons-Hawking type [5]. We examine the propagation of axial low-energy modes in expanding, strongly elongated condensates, and find that the spectrum consists of one massless (phonon) mode and a sequence of massive excitations, moving in an effective curved $1+1 \mathrm{D}$ space-time. It is demonstrated that a $1+1 \mathrm{D}$ version of the de Sitter metric [3] can be realized by the massless phonon mode in a linearly expanding Bose-Einstein condensate with constant particle interaction. We show that an atomic quantum dot (AQD) [13], placed at the center of the cloud, can be used to measure the Gibbons-Hawking quantum process. The detector's coupling to the superfluid is constructed in such a way that the natural time interval of the detector is equal to the time interval in the de Sitter metric. Therefore, the detector inside the expanding condensate is capable of measuring a thermal state at the de Sitter tempera- ture. The Gibbons-Hawking effect is a curved space-time generalization of the Unruh-Davies effect, in which a constantly accelerated detector in vacuum responds as if it were placed in a thermal bath with temperature proportional to its acceleration 14, 15. Therefore, our proposal represents a means to confirm the observer dependence of the particle content of a quantum field in curved spacetime [16].

Our approach is based on the by now well established identity of the action of a massless scalar field propagating on a curved space-time background in $D+1$ dimensions and the action of the phase fluctuations $\Phi$ in a moving inhomogeneous superfluid [17, 18, 19] (we set $\hbar=m=1$, where $m$ is the mass of a superfluid particle):

$$
\begin{aligned}
S & =\int d^{D+1} x \frac{1}{2 g}\left[-(\dot{\Phi}-\boldsymbol{v} \cdot \nabla \Phi)^{2}+c^{2}(\nabla \Phi)^{2}\right] \\
& \equiv \frac{1}{2} \int d^{D+1} x \sqrt{-\mathrm{g}} \mathrm{g}^{\mu \nu} \partial_{\mu} \Phi \partial_{\nu} \Phi .
\end{aligned}
$$

Here, $\boldsymbol{v}(\boldsymbol{x}, t)$ is the superfluid background velocity, $c(\boldsymbol{x}, t)=\sqrt{g \rho_{0}}$ the velocity of sound, $\rho_{0}(\boldsymbol{x}, t)$ the background density, and $g=4 \pi a_{s}$ is the coupling constant describing the short-range interaction between the particles in the superfluid; $a_{s}$ is the $s$-wave scattering length. In the second line of (10), the conventional hydrodynamic action is identified with the action of a minimally coupled scalar field in an effective curved space-time. In the following, we derive the effective 1+1D action of the form (11) for the lowest axial excitations of an elongated condensate from the full 3D dynamics of hydrodynamic excitations of the cigar-shaped condensate, and identify the metric tensor $\mathrm{g}_{\mu \nu}$.

We consider a large condensate confined in a strongly anisotropic harmonic trap, characterized by the frequencies $\omega_{\|}$and $\omega_{\perp}$ in the axial and radial directions, respectively $\left(\omega_{\perp} \gg \omega_{\|}\right)$. The initial condensate density is given by the usual Thomas-Fermi (TF) expression $\left|\Psi_{\mathrm{TF}}\right|^{2}=\rho_{m}\left(1-r^{2} / R_{\perp}^{2}-z^{2} / R_{\|}^{2}\right)$. Here, $\rho_{m}$ is the maximum initial density and the squared TF radii are $R_{\|}^{2}=2 \mu / \omega_{\|}^{2}$ and $R_{\perp}^{2}=2 \mu / \omega_{\perp}^{2}$, where $\mu=\rho_{m} g$ is the (initial) chemical potential. According to [20], the temporal evolution of a Bose-Einstein-condensed atom cloud under variation of the trapping frequencies is described 


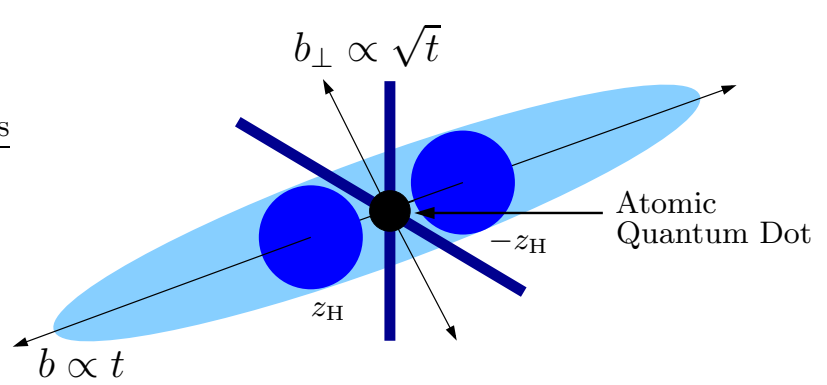

FIG. 1: Expansion of a cigar-shaped Bose-Einstein condensate. The stationary horizon surfaces are located at $\pm z_{\mathrm{H}}$, respectively. The thick dark lines represent lasers creating an optical potential well in the center.

by the scaling condensate wave function

$$
\Psi\left(r / b_{\perp}, z / b\right)=\left(\rho_{0}+\delta \rho\right)^{1 / 2} \exp \left[i\left(\Phi_{0}+\Phi\right)\right] .
$$

Here, $\Phi_{0}=-\int g \rho_{0}(0, t) d t+\dot{b} z^{2} / 2 b+\dot{b}_{\perp} r^{2} / 2 b_{\perp}$ is the background phase, where $b_{\perp}(t)$ and $b(t)$ are the scaling parameters describing the condensate expansion in the radial $(\hat{r})$ and axial $(\hat{z})$ directions, cf. Fig. The meanfield condensate density $\rho_{0}=\left|\Psi_{\mathrm{TF}}\right|^{2}\left(r / b_{\perp}, z / b\right) /\left(b_{\perp}^{2} b\right)$ contains the scaling volume $b_{\perp}^{2} b$, and $\delta \rho$ are the fluctuations of the density around mean-field.

The elementary excitations above the ground state of the BEC in the limit $\omega_{\perp} / \omega_{\|} \rightarrow \infty$ were first studied in 21]. The description of the modes is based on an adiabatic separation for the axial and longitudinal variables of the phase fluctuation field:

$$
\Phi(r, z, t)=\sum_{n} \phi_{n}(r) \chi_{n}(z, t)
$$

where $\phi_{n}(r)$ is the radial wavefunction characterized by the quantum number $n$ (we consider only zero angular momentum modes). For long wavelength axial excitations, the dispersion relation reads 21]

$$
\epsilon_{n k}^{2}=2 \omega_{\perp}^{2} n(n+1)+c_{0}^{2} k^{2},
$$

where $c_{0}=\sqrt{\mu / 2}$ and $k$ is the axial wavenumber $\left(k R_{\perp} \ll\right.$ 1). In the action (11), we use Eq. (3), with the rescaled radial wavefunction $\phi_{n} \equiv \phi_{n}\left(r / b_{\perp}\right)$, and integrate over the radial coordinate. We then find the following effective action for the axial modes of a given radial quantum number $n$,

$$
\begin{aligned}
& S_{n}=\int d t d z \frac{b_{\perp}^{2} C_{n}}{2 g}\left[-\left(\dot{\chi}_{n}-v_{z} \partial_{z} \chi_{n}\right)^{2}\right. \\
& \left.+\bar{c}_{n}^{2}\left(\partial_{z} \chi_{n}\right)^{2}+M_{n}^{2} \chi_{n}^{2}\right] .
\end{aligned}
$$

Here, the common factor $C_{n}(z)$ is given by $b_{\perp}^{2} C_{n}(z)=$ $\int_{r<r_{m}} d^{2} r \phi_{n}^{2}$, the averaged speed of sound is $\bar{c}_{n}^{2}(z, t)=$ $g C_{n}{ }^{-1} b_{\perp}^{-2} \int_{r<r_{m}} d^{2} r \rho_{0} \phi_{n}^{2}$, and the effective mass term $M_{n}^{2}(z, t)=g C_{n}{ }^{-1} b_{\perp}^{-2} \int_{r<r_{m}} d^{2} r \rho_{0}\left[\partial_{r} \phi_{n}\right]^{2} ;$ the radial cigar size is given by $r_{m}^{2}=R_{\perp}^{2} b_{\perp}^{2}\left(1-z^{2} / R_{\|}^{2} b^{2}\right)$. The phonon branch of the excitations corresponds to the $n=0$ solution of Eq. (4), for which the radial wavefunction $\phi_{0}=$ const. 21]; hence the mass term $M_{0}=0$ and $C_{0}(0)=\pi R_{\perp}^{2}, \bar{c}(0, t) \equiv \bar{c}_{n=0}(0, t)=c_{0} /\left(b_{\perp} b^{1 / 2}\right)$.

The action (5) can be identified with the action of a minimally coupled scalar field in $1+1 \mathrm{D}$, close to the center of the condensate. The line element reads 17, 18]

$$
d s^{2}=A_{c}\left[-\left(c^{2}-v_{z}^{2}\right) d t^{2}-2 v_{z} d t d z+d z^{2}\right],
$$

where $A_{c}$ is some arbitrary (space and time dependent) conformal factor and we set $c=\bar{c}(z=0, t)$. The actions (11) and (5) can be made consistent if we renormalize the phase field according to $\Phi=Z \tilde{\Phi}$ and require that $Z^{2} b_{\perp}^{2} C_{0}(0) / g=1 / \bar{c}(0)$, leading to

$$
B \equiv \frac{b_{\perp}}{b^{1 / 2}}=8 \sqrt{\frac{\pi}{2}} \frac{1}{Z^{2}}\left(\rho_{m} a_{s}^{3}\right)^{1 / 2}\left(\frac{\omega_{\perp}}{\mu}\right)^{2}=\text { const. }
$$

The constant factor $Z$ in the above relations does not influence the equation of motion, and corresponds to a renormalization of the field amplitude, $\Phi=Z \tilde{\Phi}$, which determines the coupling of $\tilde{\Phi}$ to a detector $[22$.

We require that the space-time metric is, in appropriately chosen coordinates, identical to that of a $1+1 \mathrm{D}$ de Sitter universe. We first apply the transformation $c_{0} d \tilde{t}=c(t) d t$, connecting the laboratory time $t$ to the time variable $\tilde{t}$. Defining $v_{z} / c=\sqrt{\Lambda} z=\left(B \dot{b} / c_{0}\right) z$ (note that the "dot" on $b$ and other quantities always refers to ordinary laboratory time), this results, up to the conformal factor $A_{c}$, in the line element $d s^{2}=-c_{0}^{2}\left(1-\Lambda z^{2}\right) d \tilde{t}^{2}-$ $2 c_{0} z \sqrt{\Lambda} d \tilde{t} d z+d z^{2}$. We then employ a second transformation $c_{0} d \tau=c_{0} d \tilde{t}+z \sqrt{\Lambda} d z /\left(1-\Lambda z^{2}\right)$, with a time independent $\Lambda$, to obtain the $1+1 \mathrm{D}$ de Sitter metric [3, 5]

$$
d s^{2}=-c_{0}^{2}\left(1-\Lambda z^{2}\right) d \tau^{2}+\left(1-\Lambda z^{2}\right)^{-1} d z^{2} .
$$

The quantity $\Lambda=B^{2} \dot{b}^{2} / c_{0}^{2}$ is a "cosmological constant," provided $\dot{b}$ does not depend on $t$. The transformation between $t$ and the de Sitter time $\tau$, at a given coordinate $z$, then involves the exponential "acceleration," $t / t_{0}=\exp [B \dot{b} \tau]$, where $t_{0} \sim 2 \pi / \omega_{\|}$is set by the initial conditions. The (conformally invariant) temperature associated with the effective metric (8) is the GibbonsHawking temperature [5]

$$
T_{\mathrm{dS}}=\frac{c_{0}}{2 \pi} \sqrt{\Lambda}=\frac{B}{2 \pi} \dot{b},
$$

and the horizon(s) are located at $z= \pm z_{\mathrm{H}}=$ $\pm R_{\|}\left(\omega_{\|}^{2} / 2 \mu \Lambda\right)^{1 / 2}$. Combining the latter relation with (9), we see that $z_{\mathrm{H}} / R_{\|} \ll 1$, if $T_{\mathrm{dS}} \gg \omega_{\|} / 4 \pi$. We are thus justified in neglecting the $z$ dependence in $C_{0}$ and $\bar{c}$ to describe the physics inside the horizon surfaces. 
According to Eq. (55), the equation of motion $\delta S_{0} / \delta \chi_{0}=0$ is given, at constant $B$, by

$$
B^{2} b^{2} \frac{d}{d t}\left(b^{2} \frac{d}{d t} \chi_{0}\right)-\frac{1}{C_{0}\left(z_{b}\right)} \partial_{z_{b}}\left[\bar{c}^{2}\left(z_{b}\right) C_{0}\left(z_{b}\right) \partial_{z_{b}} \chi_{0}\right]=0,
$$

where $z_{b}=z / b$ is the scaling coordinate. Apart from the factor $C_{0}\left(z_{b}\right)$, stemming from averaging over the perpendicular direction, this equation corresponds to the hydrodynamic equation of phase fluctuations in inhomogeneous superfluids [23].

At $t \rightarrow-\infty$, the condensate is in equilibrium and the quantum vacuum phase fluctuations close to the center of the condensate can be written in the following form

$$
\hat{\Phi} \equiv \hat{\chi}_{0}=\sum_{k} \sqrt{\frac{g}{4 C_{0}(0) R_{\|} \epsilon_{0 k}}} \hat{a}_{k} e^{-i \epsilon_{0 k} t+i k z}+\text { H.c. }
$$

where $\hat{a}_{k}, \hat{a}_{k}^{\dagger}$ are the annihilation and creation operators of a phonon. The intial quantum state of phonons, which we call the "adiabatic vacuum," is the ground state of the superfluid and is annihilated by the operators $\hat{a}_{k}$ (this definition of vacuum and excitations corresponds to the "adiabatic basis" of 15]). Assuming that the expansion is switched on adiabatically, the density fluctuation operator, which is conjugate to $\hat{\chi}_{0}$, then reads

$$
\begin{aligned}
\delta \hat{\rho}= & \sum_{k} \sqrt{\frac{\epsilon_{0 k}}{4 C_{0}(0) R_{\|} g}} \\
& \times \frac{d}{d t}\left\{\hat{a}_{k} \exp \left[-i \int^{t} \frac{d t^{\prime} \epsilon_{0 k}}{B b^{2}}+i k z_{b}\right]\right\}+\text { H.c. }(12)
\end{aligned}
$$

The above equation completely characterizes the $n=0$ evolution of the condensate density fluctuations.

The particle content of a quantum field state in curved space-time depends on the observer 16. We now show that the de Sitter time interval $d \tau=d t / b B=d t / b^{1 / 2} b_{\perp}$ can be effectively measured by an Atomic Quantum Dot 13. Here, the AQD is an effective two-level system with a time-dependent level splitting (see Fig.(2). It can be made in a gas of atoms possessing two hyperfine ground states $\alpha$ and $\beta$. The atoms in the state $\alpha$ represent the superfluid cigar, and are used to model the expanding universe. The AQD itself is formed by trapping atoms in the state $\beta$ in a tightly confining optical potential $V_{\mathrm{opt}}$. The interaction of atoms in the two internal levels is described by a set of coupling parameters $g_{c d}=4 \pi a_{c d}(c, d=\{\alpha, \beta\})$, where $a_{c d}$ are the $s$-wave scattering lengths characterizing short-range intra- and inter-species collisions; $g_{\alpha \alpha} \equiv g, a_{\alpha \alpha} \equiv a_{s}$. The on-site repulsion between the atoms $\beta$ in the $\operatorname{dot}$ is $U \sim g_{\beta \beta} / l^{3}$, where $l$ is the characteristic size of the ground state wavefunction of atoms $\beta$ localized in $V_{\text {opt }}$. In the following we consider the collisional blockade limit of large $U>0$, where only one atom of type $\beta$ can be trapped in the dot. This assumes that $U$ is larger than all other relevant frequency scales in the dynamics of both the AQD

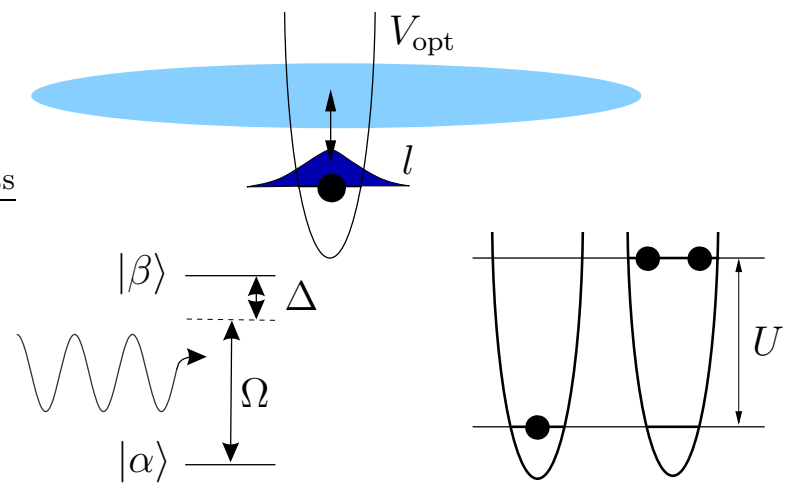

FIG. 2: Level scheme of the "Atomic Quantum Dot," which is embedded in the superfluid cigar, and created by an optical well for atoms of a hyperfine species different from that of the condensate. Double occupation of the dot is prevented by a collisional blockade mechanism.

and the expanding superfluid. (The value of $g_{\beta \beta}$ may be increased using Feshbach resonances [24].) As a result, the appropriate collective coordinate of the AQD is modeled by a pseudo-spin- $1 / 2$, with spin-up/spin-down state corresponding to occupation by a single/no atom in state $\beta$.

Conversion of atoms between the states $\alpha$ and $\beta$ is caused by a laser-driven transition characterized by Rabi frequency $\Omega$ and detuning $\Delta$. The Hamiltonian of the AQD interacting with the superfluid is

$$
\begin{aligned}
H_{\mathrm{AQD}}= & \left\{-\Delta+g_{\alpha \beta}\left[\rho_{0}(0, t)+\delta \rho(0, t)\right]\right\} \frac{\left(1+\sigma_{z}\right)}{2}(13) \\
& +\Omega\left(\rho_{0}(0, t) l^{3}\right)^{1 / 2} \exp \left[i\left(\Phi+\Phi_{0}\right)\right] \sigma_{+}+\text {H.c. }
\end{aligned}
$$

where we use Pauli matrix notation, i.e. $\sigma_{+}=\sigma_{x}+i \sigma_{y}$. We introduce the wavefunction of the AQD in the form $\psi=\psi_{\beta} \exp \left[i\left(\Phi+\Phi_{0}\right)\right]|\beta\rangle+\psi_{\alpha}|\alpha\rangle$. Using the scaling expression (2) for the condensate wave function, we find

$$
\begin{aligned}
i \frac{d \psi_{\beta}}{d \tau} & =\left\{b B\left[-\Delta+\rho_{0}\left(g_{\alpha \beta}-g\right)\right]+\delta V\right\} \psi_{\beta}+\frac{\omega_{0}}{2} \psi_{\alpha} \\
i \frac{d \psi_{\alpha}}{d \tau} & =\frac{\omega_{0}}{2} \psi_{\beta}
\end{aligned}
$$

where $\tau$ is the de Sitter time, and $\omega_{0}=2 \Omega\left(\rho_{m} l^{3}\right)^{1 / 2}$ is independent of $\tau$. The perturbation potential

$$
\delta V(\tau)=\left(g_{\alpha \beta}-g\right) B b(\tau) \delta \rho(0, \tau)
$$

provides the coupling between the AQD and the expanding superfluid (if $g_{\alpha \beta} \simeq g$, higher order terms in the density fluctuations have to be taken into account in the Rabi term of (13) ). We suggest to operate the detector at $\Delta(t)=\left(g_{\alpha \beta}-g\right) \rho_{0}(0, t)=\left(g_{\alpha \beta}-g\right) \rho_{m} /\left(\dot{b}^{2} B^{2} t^{2}\right)$, so that in zeroth order in $\delta V$ we obtain an effective twolevel system with the level splitting $\omega_{0}$, which plays the 
role of a frequency standard of the detector. By adjusting the laser intensity contained in the Rabi frequency $\Omega$, one can change $\omega_{0}$, and thus probe the response of the detector for various frequencies.

The detector response can be calculated using the twopoint correlation function of the coupling operator $\delta \hat{V}$. The probabilities for the detector to "click," corresponding to excitation $\left(P_{+}\right)$and de-excitation $\left(P_{-}\right)$of the detector, per unit of de Sitter time, are given by [14, 15]:

$$
\frac{d P_{ \pm}}{d \tau}=\lim _{\mathcal{T} \rightarrow \infty} \frac{1}{\mathcal{T}} \int^{\mathcal{T}} \int^{\mathcal{T}} d \tau d \tau^{\prime}\left\langle\delta \hat{V}(\tau) \delta \hat{V}\left(\tau^{\prime}\right)\right\rangle e^{\mp i \omega_{0}\left(\tau-\tau^{\prime}\right)}
$$

Using the explicit form of the detector-to-field coupling (15), we obtain

$$
\left\langle\delta \hat{V}(\tau) \delta \hat{V}\left(\tau^{\prime}\right)\right\rangle \propto\left(\rho_{m} a_{s}^{3}\right)^{1 / 2}\left(\frac{\omega_{\perp}}{\mu}\right)^{2} \frac{B^{2}\left(g_{\alpha \beta} / g-1\right)^{2} T_{\mathrm{dS}}^{2}}{\sinh ^{2}\left[\pi T_{\mathrm{dS}}\left(\tau-\tau^{\prime}\right)\right]} .
$$

This correlation function is proportional to one characterizing a thermal phonon state at a temperature $T_{\mathrm{dS}}$ in a condensate at rest. This means that our de Sitter detector at the center of the condensate responds to the scaling vacuum as if it were placed in a thermal bath at a temperature $T_{\mathrm{dS}}$. Indeed, substituting the correlator (17) into Eq. (16) we find that the quantities $d P_{ \pm} / d \tau$ at late times are time independent, and satisfy detailed balance conditions corresponding to thermal equilibrium at the de Sitter temperature given in Eq. (9):

$$
\frac{d P_{+} / d \tau}{d P_{-} / d \tau}=\frac{n_{\mathrm{B}}}{1+n_{\mathrm{B}}}
$$

where $n_{\mathrm{B}}=\left(\exp \left[\omega_{0} / T_{\mathrm{dS}}\right]-1\right)^{-1}$ is the Bose distribution function. We stress that the AQD observes a thermal spectrum, even though the quantum state of the fluctuations is adiabatically connected to the initial vacuum state, i.e., no excitations in the adiabatic basis characterized by the $\hat{a}_{k}$ are created (note that the time interval of the adiabatic vacuum is $\left.d t / B b^{2}=d \tau / b\right)$. The latter process corresponds to "cosmological" quasiparticle production 11, 12], which can be neglected in our situation.

Our experimental proposal consists in the following steps: (a) Preparation of a large TF condensate at very low temperatures. (b) Introduction of an AQD at the center (respectively an array of AQDs to increase the signal), which can be used to determine the initial temperature of the cloud. (c) Linear axial expansion of the condensate according to (7). The Rabi oscillations of the AQD can be used to monitor the thermal distribution $n_{\mathrm{B}}$ in (18). Since $Z^{2} \propto\left(\rho_{m} a_{s}^{3}\right)^{1 / 2}\left(\omega_{\perp} / \mu\right)^{2}$ and $P_{ \pm} \propto Z^{2}$ [22], the initial condensate has to be quite dense and close to the quasi-1D régime. The particle density throughout the cloud decreases like $t^{-2}$. Therefore, the rate of threebody losses quickly decreases during expansion, and comparatively long observation times are feasible.
The AQD as a detector is suitable to measure the de Sitter time interval, because it couples linearly to the square root of the density of the superfluid gas. In principle, one can construct detectors coupling to different powers of density or superfluid velocity, and then more generally experimentally study the non-uniqueness of the particle content of various quantum states in curved space-time [16]. For example, outcoupling pairs of atoms by photoassociation is a means to set up a detector which has $d \tau / d t \propto \rho_{0}$. Finally, we note that while in this paper we have studied only the $n=0$ massless axial phonon modes, strongly elongated condensates can also be used to study the evolution of massive bosonic excitations. Together with a natural Planck scale $E_{\text {Planck }} \sim \mu$, this provides the opportunity to investigate, on a laboratory scale, the influence of finite quasiparticle mass and the trans-Planckian spectrum on the propagation of relativistic quantum fields in curved space-time.

We thank E.A. Cornell for an inspiring discussion on the experimental feasibility of our theoretical ideas. We acknowledge helpful discussions with R. Parentani, R. Schützhold, G. E. Volovik, P. Zoller, and A. Recati. P. O.F. has been supported by the Austrian FWF and the Russian RFRR, and U.R.F. by the FWF. We gratefully acknowledge support from the ESF Programme "Cosmology in the Laboratory."

[1] G. R. Pickett et al., Nature 383, 570 (1996).

[2] G. E. Volovik, The Universe in a Helium Droplet (Oxford University Press, Oxford, 2003).

[3] W. de Sitter, Mon. Not. R. Astron. Soc. 78, 3 (1917).

[4] A. Linde, Inflation, Quantum Cosmology and the Anthropic Principle, hep-th/0211048

[5] G. W. Gibbons and S.W. Hawking, Phys. Rev. D 15, 2738 (1977).

[6] J. R. Anglin and W. Ketterle, Nature 416, 211 (2002).

[7] W. G. Unruh and R. Schützhold, Phys. Rev. D 68, 024008 (2003).

[8] L. J. Garay, J. R. Anglin, J. I. Cirac, and P. Zoller, Phys. Rev. Lett. 85, 4643 (2000).

[9] C. Barceló, S. Liberati, and M. Visser, Class. Quantum Grav. 18, 1137 (2001).

[10] U. Leonhardt, T. Kiss, and P. Öhberg, J. Opt. B 5, S42 (2003).

[11] P. O. Fedichev and U. R. Fischer, cond-mat/0303063

[12] C. Barceló, S. Liberati, and M. Visser, gr-qc/0305061

[13] A. Recati et al., cond-mat/0212413

[14] W. G. Unruh, Phys. Rev. D 14, 870 (1976).

[15] N.D. Birrell and P. C. W. Davies, Quantum Fields in Curved Space (Cambridge University Press, Cambridge, England, 1984).

[16] S. A. Fulling, Phys. Rev. D 7, 2850 (1973).

[17] W. G. Unruh, Phys. Rev. Lett. 46, 1351 (1981).

[18] M. Visser, Class. Quantum Grav. 15, 1767 (1998).

[19] U. R. Fischer and M. Visser, Ann. Phys. (N.Y.) 304, 22 (2003); Phys. Rev. Lett. 88, 110201 (2002).

[20] Yu. Kagan, E. L. Surkov, and G. V. Shlyapnikov, Phys. 
Rev. A 54, R1753 (1996); Y. Castin and R. Dum, Phys. Rev. Lett. 77, 5315 (1996).

[21] E. Zaremba, Phys. Rev. A 57, 518 (1998).

[22] P. O. Fedichev and U. R. Fischer, cond-mat/0307200
[23] S. Stringari, Phys. Rev. Lett. 77, 2360 (1996).

[24] A. Marte et al., Phys. Rev. Lett. 89, 283202 (2002). 